\title{
Genome-wide identification of the auxin response factor gene family in Cicer arietinum
}

\author{
Jose V. Die ${ }^{*} \mathbb{D}$, Juan Gil and Teresa Millan
}

\begin{abstract}
Background: Auxin Response Factors act as critical components of the auxin-signaling pathway by regulating the transcription of auxin-responsive genes. The release of the chickpea reference genome provides an opportunity to identify and characterize the ARF gene family in this important legume by a data mining coupled by comparative genomics approaches.

Results: We performed a comprehensive characterization and analysis of 24 ARF genes in the chickpea reference genome. Comparative phylogenetic analysis of the ARF from chickpea, Medicago and Arabidopsis suggests that recent duplications have played a very limited role in the expansion of the ARF chickpea family. Gene structure analysis based on exon-intron organization provides additional evidence to support the evolutionary relationship among the ARF members. Conserved motif analysis shows that most of the proteins fit into the canonical ARF structure model, but 9 proteins lack or have a truncated dimerization domain. The mechanisms underlying the diversification of the ARF gene family are based on duplications, variations in domain organization and alternative splicing. Concerning duplications, segmental, but not tandem duplications, have contributed to the expansion of the gene family. Moreover, the duplicated pair genes have evolved mainly under the influence of purifying selection pressure with restricted functional divergence. Expression profiles responding to various environmental stimuli show a close relationship between tissue and expression patterns. Promoter sequence analysis reveals an enrichment of several cis-regulatory elements related to symbiosis, and modulation of plant gene expression during the interaction with microbes.

Conclusions: In conclusion, this study provides a comprehensive overview of the ARF gene family in chickpea. Globally, our data supports that auxin signaling pathway regulates a wide range of physiological processes and stress responses. Our findings could further provide new insights into the complexity of the regulation of ARF at the transcription level that may be useful to develop rational chickpea breeding strategies to improve development or stress responses. Our study also provides a foundation for comparative genomic analyses and a framework to trace the dynamic evolution of ARF genes on a large time-scale within the legume family.
\end{abstract}

Keywords: ARF, Bioinformatics, Chickpea, Gene duplication, Gene expression, RT-qPCR, Rstat

\footnotetext{
*Correspondence: jodiera@upv.es; jodiera@alumni.upv.es; jose.die@uco.es

Department of Genetics, ETSIAM, University of Córdoba, Córdoba, Spain

(c) The Author(s). 2018 Open Access This article is distributed under the terms of the Creative Commons Attribution 4.0 International License (http://creativecommons.org/licenses/by/4.0/), which permits unrestricted use, distribution, and reproduction in any medium, provided you give appropriate credit to the original author(s) and the source, provide a link to the Creative Commons license, and indicate if changes were made. The Creative Commons Public Domain Dedication waiver (http://creativecommons.org/publicdomain/zero/1.0/) applies to the data made available in this article, unless otherwise stated.
} 


\section{Background}

The plant hormone auxin (indole-3-acetic acid) is a key regulator of virtually every aspect of plant growth and development. Most of these processes are initiated or mediated through auxin-regulated gene expression, which in turn is controlled by proteins belonging three gene families: receptors (F-box), repressors (Auxin/Indole-3-Acetic Acid; Aux/IAA) and auxin response factor proteins (ARF). ARF represent the core of auxin signaling [1]. In the last few years, a considerable amount of new information has appeared on the regulation of ARF gene expression, target genes controlled by ARF, and the mechanisms by which ARF regulate those target genes. According to transient assays and sequence analysis, ARF proteins are divided into transcriptional activators and repressors [2]. ARF activators are transcription factors that mediate auxin-dependent transcriptional regulation by binding to auxin-response elements in the promoters of auxin-inducible genes in a dose-dependent manner [3]. In the absence of auxin, Aux/IAA proteins prevent ARF-mediated transcription by forming heterodimers with ARF activators $[4,5]$. In the presence of auxin, Aux/IAA proteins are targeted to the $26 \mathrm{~S}$ proteasome, which can be hypothesized to release interacting ARF activators from inhibition [6]. Contrary to ARF activators, ARF repressors have very limited interactions with other ARF and Aux/IAA proteins [5]. Though some auxin responses occur throughout the plant, others depend on the actual developmental context, conferring the tissue-specific response to auxin. Those responses involve the action of specific pairs of ARF and Aux/IAA proteins $[7,8]$. Concerning the specificity, the domain architecture of ARF proteins plays an important role. Most ARF consist of an N-terminal DNA-binding domain (DBD), a variable middle region (MR) and a carboy-terminal dimerization domain (CTD, domains III and IV). The MR confers transcriptional activation or repression depending on its amino acid composition. Thus, the MR enriched for glutamine residues function as activation regions, while MRs serine-rich, serine and proline-rich, and serine and glycine-rich function as repression regions in Arabidopsis thaliana [9, 10]. Domains III and IV are essential for the heterodimerization between ARF and Aux/IAA proteins [11, 12]. These domains are also known to facilitate homodimerization, which appears to be required in some cases for the efficient binding of ARF to DNA [10].

In recent years, many of the major crops have been sequenced. Crop genome sequences, even at the current level of completeness have had a major impact on crop research or improvement in a relatively short time [13]. Chickpea (Cicer arietinum L.) is globally the second most important grain legume [14]. Although the chickpea yield potential has increased over the last years, the production is constrained by several major abiotic (drought, heat, high salinity) and biotic stresses (fusarium wilt, ascochyta blight) $[15,16]$. Genomic resources represent the starting point for understanding the unique traits present in a given crop and are also tools for implementation of molecular breeding for the development of improved varieties [17]. Until recently, lack of information on legume genomes traditionally restricted the genome-wide survey of genes in response to the environment or stresses. Fortunately, the genome sequence of chickpea has become available in the last few years $[18,19]$. Chickpea genome sequences provide an unprecedented resource, which can be exploited in numerous ways.

As a central role of the auxin-signaling pathway, the ARF multigene family is present in all major divisions of land plants [6]. The ARF family has been characterized in both annual herbaceous plants and woody perennials. Gene member numbers are variable between species ranging from 18 in peach [20] to 51 members in soybean [21]. Considering the important role of ARF family members as regulators of plant growth and developmental processes in other plant species, it is important to explore this gene family in chickpea. In this work, we provide comprehensive information on the genomic structures, chromosomal locations, sequences homology, evolutionary duplication history, cis-regulatory elements and expression profiles of 24 ARF genes in C. arietinum.

\section{Methods}

\section{Genome-wide survey of ARF genes in C. arietinum}

Comprehensive identification of $C$. arietinum ARF gene family members was achieved using Medicago truncatula ARF proteins. The M. truncatula ARF protein sequences were downloaded from the Phytozome v12.0 database (http://www.phytozome.net) and used as queries in BLASTP searches [22] to identify the corresponding ARF gene members in the chickpea proteome using a cut-off of $30 \%$ identity, $30 \%$ query coverage and evalue $<1.0 \mathrm{E}-10$. For validation, we also used Arabidopsis ARF proteins as queries following the same procedure. The hidden Markov model (HMM) profiles of the ARF gene family [Pfam 02309: AUX/IAA family; Pfam 06507: ARF (AUX_RESP); Pfam 02362: B3 DNA binding domain (B3)] were used to confirm the identity of the candidate chickpea ARF genes. The domains of all obtained ARF were further confirmed as well by using the NCBI Conserved Domain Database (CDD, https://www.ncbi. nlm.nih.gov/cdd/) and e-value of 0.01 [23]. For exhaustive identification of divergent chickpea gene family members, we used the chickpea ARF proteins as queries in BLASTP searches against the chickpea proteome. In order to check for any possible non-predicted gene, we run tBLASTn searches against the $C$. arietinum $\mathrm{CDC}$ 
Frontier genome assembly v1 (ASM33114v1 assembly, https://www.ncbi.nlm.nih.gov/assembly/GCF_000331145 $.1 /$ ). All that process enabled us to obtain 45 unique ARF protein sequences. Using one gene model per locus, we identified 24 C. arietinum non-redundant ARF genes. Information on chromosomal location, locus ID, amino acid length, molecular weight and number of exons was retrieved from the NCBI using custom $\mathrm{R}$ scripts. The Compute pI tool on the ExPASy proteomics server database (http://web.expasy.org/compute_pi/) was used to predict the theoretical isoelectric point $(\mathrm{pI})$ of each CaARF protein.

\section{Sequence alignment, prediction of amino-acid content, and protein classification}

Multiple sequence alignments were conducted on the full length of the 24 ARF protein sequences using the default parameters of the MUSCLE program [24]. Amino acid content of the MR domain in CPARF was calculated using the 'Biostrings' $\mathrm{R}$ package (version 2.42; [25]). The classification of CPARF was based on the respective amino acid content [Domains with CTD: Glutamine/serine/leucine (QSL)-rich MR; Repressor with a carboxyl terminal domain (CTD); Serine/proline/ glycine/leucine (SPGL)-rich MR; Repressor without CTD: Glycine-rich MR].

\section{Phylogenetic analysis and gene structure}

The evolutionary history was inferred using the Neighbor-Joining method [26]. The bootstrap consensus tree inferred from 1000 replicates [27]. The evolutionary distances were computed using the Poisson correction method [28] and are in the units of the number of amino acid substitutions per site. Evolutionary analyses were conducted in MEGA5 [29]. The exon/intron structure of the chickpea ARF genes was based on the genome and coding sequences and was identified using the GSDS software (http://gsds.cbi.pku.edu.cn/; [30]).

\section{Gene duplication analysis}

Duplication analysis for CaARF genes was performed using Plant Genome Duplication Database (PGDD; http://chibba.agtec.uga.edu/duplication/; [31]). Circoletto tool was used to determine and plot sequence similarity [32]. To define a tandem cluster, the following parameters were established: a cluster should contain at least two genes; a sliding window size should be $<250 \mathrm{~kb}$ [33]. The number of nonsynonymous substitutions per nonsynonymous site (Ka) and synonymous substitution per synonymous site $(\mathrm{Ks})$ values were extracted from PGDD. The Ks values obtained for each gene pair were then translated into divergence time in millions of years assuming a rate of $6.1 \times 10-9$ substitutions per site per year for eudicots [34]. The divergence time $(\mathrm{T})$ was calculated as $\mathrm{T}=\mathrm{Ks} /$ 2GAMMA (GAMMA = 6.1 × 10-9; [35]).

\section{In silico expression analysis}

The coding sequences of ARF genes were employed to query the NCBI chickpea ESTs. Searching parameters were set as follows: megablast, identity >95\%, length $>200 \mathrm{bp}$ and E-value $<10-10$.

\section{Plant material}

Plant material and treatments have been described in detail elsewhere [36]. Briefly, chickpea plants were grown in a growth chamber $\left(12 \mathrm{~h}\right.$ of light at $25^{\circ} \mathrm{C}$ and $12 \mathrm{~h}$ of dark at $22{ }^{\circ} \mathrm{C}$ ) until the moment that stress treatments started. Two weeks after flowering, some plants were exposed to cold and drought treatments. For cold treatment, a pool was exposed to a cycle of $12 \mathrm{~h}$ day/12 h night at temperatures of $25{ }^{\circ} \mathrm{C}$ and $5{ }^{\circ} \mathrm{C}$, respectively. Leaves and flowers from the susceptible ICC4918 genotype (desi) were collected after the seventh night at $5{ }^{\circ} \mathrm{C}$. Another pool was exposed to drought conditions by allowing the $5-10 \%$ loss of their water content per day. Leaves and stems from the susceptible ILC72 genotype (kabuli) were collected when the treatment pots lost $50 \%$ of their water content. For the salinity stress, 18day-old plants were irrigated with Hoagland's nutrient medium with $150 \mathrm{mM} \mathrm{NaCl}(\mathrm{pH}$ 6.5). Leaf, stem and root tissues from the susceptible ICCV2 genotype (kabuli) were collected $24 \mathrm{~h}$ after treatment. Two ascochyta blight differential germplasm lines (WR315 susceptible, and ILC3279 resistant; desi and kabuli, respectively) were inoculated with a highly virulent isolate of Ascochyta rabiei. Spore suspensions at concentrations of $5 \times$ $10^{5}$ were prepared from 14-day-old fungal cultures that were grown on V8 agar at $20{ }^{\circ} \mathrm{C}$ and $12 \mathrm{~h}$ light/dark. Inoculations were performed on two-week-old plants by spraying approximately $5 \mathrm{ml}$ of the spore suspension on each plant. The inoculated plants were incubated in the dark at $20{ }^{\circ} \mathrm{C}$ and $100 \%$ continuous relative humidity for $24 \mathrm{~h}$ to facilitate infection. Plants were then placed in a growth chamber that was set to cycle at $12 \mathrm{~h}$ day $\left(20^{\circ} \mathrm{C}\right)$ and $12 \mathrm{~h}$ night $\left(16{ }^{\circ} \mathrm{C}\right)$ and $100 \%$ relative humidity. Leaf tissues from inoculated plants were collected $72 \mathrm{~h}$ after inoculation. Control roots and leaves were separately harvested from 19-day-old plants grown in the growth chamber in $12 \mathrm{~h}$ day $\left(25^{\circ} \mathrm{C}\right) / 12 \mathrm{~h}$ night $\left(22^{\circ} \mathrm{C}\right)$. All tissues were immediately frozen in liquid nitrogen and stored at $-80{ }^{\circ} \mathrm{C}$ until RNA extraction. Each sample was made by a pool of at least five plants. Two biological repetitions were performed for each treatment.

\section{RNA extractions and quality controls}

Total RNA was isolated using the TRIZOL reagent (Invitrogen, CA, USA) according to the manufacturer's 
protocol. RNA concentration was determined by measuring the optical density at $260 \mathrm{~nm}$ using a NanoDrop ND-1000 spectrophotometer (Nanodrop Technologies, USA). RNA quality was assessed by combining information from several control steps. First, purity was inferred from the absorption ratios using the NanoDrop. Only the RNA samples with A260/A280 ratio between 1.75 and 2.1 and A260/A230 greater than 2.0 were used in the analysis. Then, we amplified segments of the $5^{\prime}$ and $3^{\prime}$ regions of a malate oxidoreductase gene across the cDNA samples by qPCR (as described below) to infer the integrity of the total RNA. After NanoDrop measurements and integrity checking, all RNA samples were adjusted to the same concentration, measured and adjusted again to homogenize RNA input in the subsequent reverse-transcription reactions.

\section{First strand CDNA synthesis and quality controls}

Total RNA $(1 \mu \mathrm{g})$ was reverse-transcribed using the QuantiTec Reverse Transcription Kit (Qiagen, Hilden, Germany), according to the manufacturer's instructions. We tested for presence of genomic DNA contamination (gDNA) by performing minus RT (-RT) controls, containing all components (including template RNA) except the reverse transcriptase. As a positive control, a quantity equivalent to that of the cDNA used as a template in the subsequent qPCRs amplification (i.e. $10 \mathrm{ng}$ of genomic DNA) was amplified using a primer pair designed from an exon of a tubulin sequence (GR913042). The cDNA samples were considered to be suitable for further analysis because no amplification was detected in any -RT control after 40 cycles. The cDNAs were diluted to a final volume of $100 \mu$ l. The efficiency of cDNA synthesis, which in turn is dependent on the intactness of mRNA (RNA integrity) was examined using a $3^{\prime}: 5^{\prime}$ amplification ratio assessment [37] to amplify cDNA fragments in the $5^{\prime}(81 \mathrm{bp})$ and $3^{\prime}(80 \mathrm{bp})$ regions of a malate oxidoreductase gene (MOR; AJ404642). The fragments are 1671 and $450 \mathrm{bp}$, respectively, from the 3' end of the cDNA. The primer sequences are MOR_5'F, 5'-CGACCGTTGTCTGATTTTGTGA-3'; MOR_5'R, 5'GGCCATTTTCAGAACCCCTAA-3'; and MOR_3'F, 5'GCTTCGAGCAGCAGTTGAAGAA-3'; and MOR_3'R, 5'-CTTTTGACATGTGTGCAAGTT-3'. The 3':5' amplification ratio of the MOR cDNA fragments was calculated using the comparative $\mathrm{Cq}$ method [38]. The average ratio was $1.51 \pm 0.11$. All ratios were $<3$.8-fold. Only if ratios were $>4$.4-fold would RNA quality be deemed inadequate [39]. Therefore, the cDNAs were judged to be suitable for qPCR analysis.

\section{Primer design and quality controls}

ARF primers were designed using the following criteria: Tm of $60 \pm 1{ }^{\circ} \mathrm{C}$ and PCR amplicon lengths of 70-95 bp, yielding primer sequences with lengths of 19-22 nucleotides and GC contents of $45-60 \%$. For primer design improvement, amplicon sequences were checked with the nucleic acid-folding software MFOLD version 3.4 software [40]. Potential formation of secondary structures were evaluated with default settings of minimal free energy, $50 \mathrm{mM} \mathrm{Na}{ }^{+}, 3 \mathrm{mM} \mathrm{Mg}^{2+}$, and an annealing temperature of $60{ }^{\circ} \mathrm{C}$. We chose primers that would yield amplicons with minimal folding structures and melting temperatures that would not hamper annealing. Designed primers were synthesized by Integrated DNA Technologies (Leuven, Belgium).

\section{Real-time qPCR assays and normalization}

Real-time qPCR reactions (RT-qPCR) were carried out in a CFX Connect Real-Time PCR Detection System thermal cycler (Bio-Rad, Hercules, CA, USA) using iTaq Universal SYBR Green supermix (Bio-Rad) to monitor dsDNA synthesis. Reactions contained $1.5 \mu \mathrm{l}$ of the diluted cDNA as a template and $0.2 \mu \mathrm{M}$ of each primer in a total volume reaction of $10 \mu \mathrm{l}$. The following standard thermal profile was used for all PCRs: polymerase activation $\left(95{ }^{\circ} \mathrm{C}\right.$ for $\left.3 \mathrm{~min}\right)$, amplification and quantification cycles repeated 40 times $\left(95{ }^{\circ} \mathrm{C}\right.$ for $3 \mathrm{~s}, 60{ }^{\circ} \mathrm{C}$ for $30 \mathrm{~s}$ ). The specificity of the primer pairs was checked by melting-curve analysis performed by the PCR machine after 40 amplification cycles $\left(60\right.$ to $\left.95{ }^{\circ} \mathrm{C}\right)$. Fluorescence was analyzed using CFX Manager Software v2.1 (BioRad). All amplification plots were analyzed using a base line threshold of 100 relative fluorescence units (RFU) to obtain $\mathrm{Cq}$ (quantification cycle) values for each genecDNA combination. To normalization of data, we evaluated the stable expression of four reference genes in our dataset. Three candidates encoding a protein phospatase protein $(P P 2 A)$, pentatricopeptide repeat-containing protein $(\mathrm{PPR})$ and ubiquitin-like protein $(U B Q)$ were selected based on previous reports [36]. We also tested the expression of a transcription factor initiation IIA (TFIIA) whose homolog in pea was one of the most stable genes under a variety of conditions [41]. Two programs were used to determine which reference genes were best suited for transcript normalization. We first used the statistical algorithm geNorm [42]. In a second approach, the coefficient of variation of normalized relative expression levels was calculated according to the formulas described in the qBase software [43]. The results indicated that PP2A and TFIIA were the most stable references in our dataset with values very inside the optimal range for heterogeneous sample panels ( $M$ and $\mathrm{CV}$ values lower than 1 and 0.5 respectively according to Hellemans et al. (2007) [43] (Additional file 1: Figure S1). Therefore, the expression of each target ARF gene was normalized to the geometric average of $P P 2 A$ and TFIIA. The overall mean real-time qPCR 
amplification efficiency of each primer pair (E) was estimated from linear regression analysis and the eq. $(1+\mathrm{E})=$ $10^{\text {slope }}$ implemented in the LinReg software [44]. Finally, the expression levels of the chickpea ARF genes were calculated using the advanced relative quantification model with efficiency correction, multiple reference gene normalization and the use of error propagation rules [43].

\section{Promoter sequence analysis}

To investigate the promoter regions of ARF genes, 1. $5 \mathrm{~kb}$ of genomic DNA sequences upstream of initiation codon ATG were retrieved from the genome assembly. Cis-regulatory elements (CREs) known to be involved in auxin responses as well as the regulation under biotic and abiotic stresses were selected for examination. Occurrence and distribution of CREs over a given promoter sequence analysis were performed using standard Python scripts. The expected frequency of each motif was calculated using the average $\mathrm{G}+\mathrm{C}$ content of $28 \%$ observed in our chickpea promoters dataset. Probabilities were estimated based on control sets (2000 Monte Carlo simulations, each set $n=23$ and $1500 \mathrm{bp}$ length). The characterized CREs included: three elements related to dehydration, high salinity and low temperature (MYCATERD1 [CATGTG], MYCATERD22 [CACATG] and ABRE [ACGTGTC]; [45]); three CREs commonly found in defensin promoters (GT1GMSCAM4 [GAAAAA], RAV1AAT [CAACA] and motif CTCTT; [46]); the element AGCBOXNPGLB [AGCCGCC], which is known to be the binding sequence of the ethylene response factor [47]; a pathogen/elicitor response element WBBOXPCWRKY1 [TTTGACY] [48]; a sugar responsive element associated with auxin responses SURE2STPAT21 [AATACAAAA] [49]); and the auxin-responsive element AuxRe1 [TGTCTC] [3].

\section{Code availability}

A custom pipeline of scripts with the open-source interface for the statistics software R, ([50]) and the opensource interface RStudio ([51], http://www.rstudio.com/) were used to retrieve data from NCBI (gene IDs, accession numbers, molecular weights, protein lengths, location on chromosomes and exon counts) and perform data analysis. $\mathrm{R}$ markdown and $\mathrm{R}$ code files used in this study are available in our git-based, publicly accessible repository (https://github.com/jdieramon/ChickpeaPro ject). We will continue to update and modify the code repository. However, older versions of the code can be retrieved using the command line-based git program. The code is distributed under the open source MIT License.

\section{Results and discussion}

Genome-wide identification and chromosomal distribution of $C$. arietinum ARF genes

The procedure to identify all members of the ARF gene family in the C. arietinum genome is shown in Fig. 1. BLASTp searches followed by HMM profile and domain analyses using the NCBI's CDD tool resulted in the primary identification of 45 potential ARF protein sequences in the chickpea genome. Subsequently, the redundant sequences with the same chromosome locations were removed from the candidate list. In the end, using one gene model per locus, a total of 24 chickpea ARF (CaARF) were extracted and named according to their locations from top to bottom on the chickpea chromosomes (from Chr. 1 to Chr. 8). This nomenclature system is broadly used in genome-wide studies and provides a unique identifier for each member of a given gene family. For example, members of the ARF family have been named that way in rice [52], maize [53], soybean [21] or grape [54]. Information on these 24 sequences (name, locus ID, length, location on chromosome and basic data about the deduced peptide) is listed in Table 1. The exon number of CaARF genes ranged from 2 (CaARF8) to 16 (CaARF12, CaARF19). The sizes of the deduced proteins varied markedly from 444 (CaARF23) to 1125 amino acid residues (CaARF20). The corresponding molecular masses varied from 50.69 to 126 . $20 \mathrm{kDa}$ and the predicted isoelectric points (pIs) varied from 5.55 (CaARF11) to 8.56 (CaARF4). As illustrated by other plant species, the wide range of pIs suggests that the chickpea ARF proteins can work in very different subcellular environments. The percentage of identity between the predicted chickpea and the Medicago ARF protein sequences ranged from $40.1 \%$ to $94.8 \%$ (Table 1). As expected, CaARF were more closely related to those from the model legume M. truncatula than those from Arabidopsis (Additional file 2: Figure S2).

CaARF gene locations were mapped on chromosomes in order to gain an insight into the organization of CaARF genes on the genome. Based on the available $C$. arietinum genome assembly, 23 out the 24 CaARF genes were distributed among seven of the eight chromosomes. We could not map CaARF24. The other 23 ARF genes were unevenly distributed through the chickpea genome. Two chromosomes (chrs. 1 and 6) contained more than $56 \%$ of the mapped ARF. The total distribution was the following: Chromosome 6 contained the highest number with 7 ARF genes (30.4\%), followed by chromosome 1 (6 genes, 26.1\%) and chromosome 7 (4 genes, 17.4\%). Chromosome 2 and 4 showed 2 genes each, whereas chromosomes 3 and 1 contained 1 gene each. Chromosome 8, which is the shortest in the chickpea genome, did not contain any ARF gene (Fig. 2 and Additional file 3: Figure S3). 


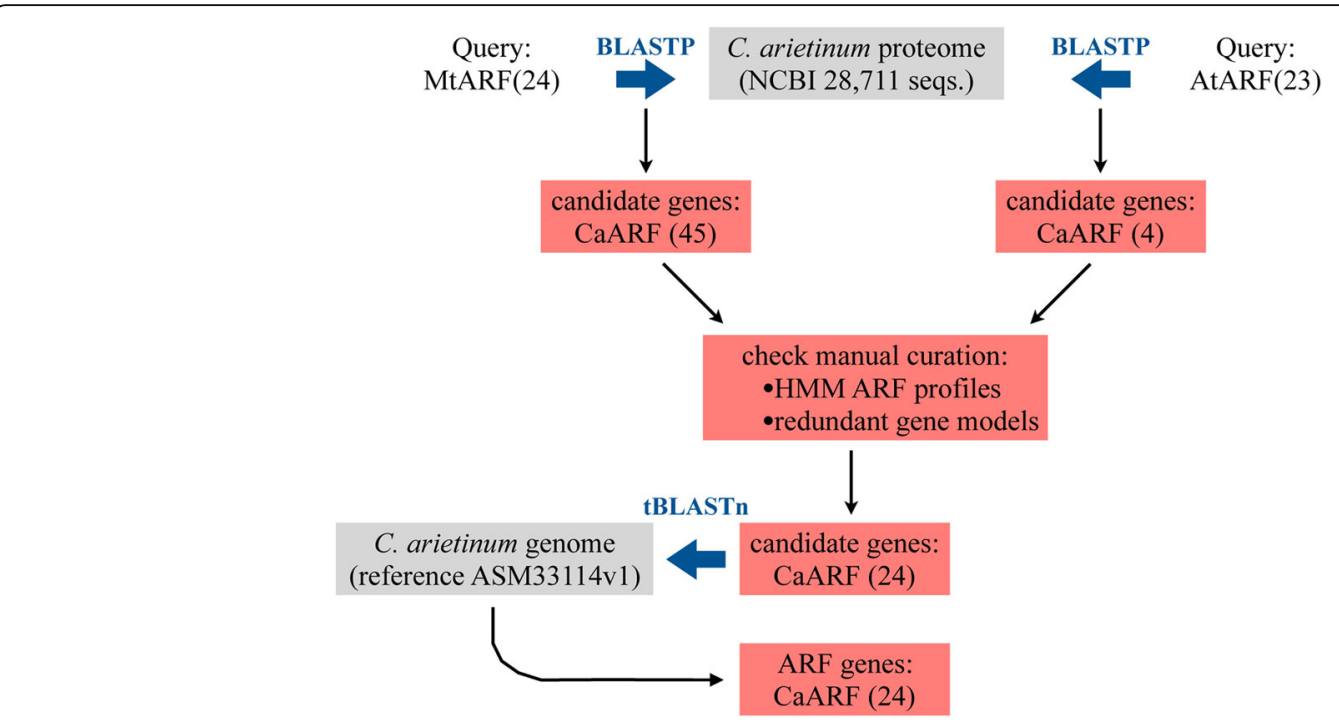

Fig. 1 Schema of the workflow to identify ARF genes in C. arietinum. Medicago ARF proteins were used as query to search for orthologs (BLASTP) in the predicted chickpea genome. The same procedure was applied using Arabidopsis ARF proteins. The output was further examined by manual curation and redundant and/or invalid genes were removed from the data set. This step resulted in 24 complete chickpea ARF protein sequences. Those candidates were blasted against the chickpea genome to identify any possible unpredicted gene

Table 1 ARF gene family in chickpea

\begin{tabular}{|c|c|c|c|c|c|c|c|c|c|c|c|}
\hline Name ID & Locus ID & Protein ID & Chr & Chr start & Chr end & Strand & Length (aa) & $\mathrm{pl}$ & Mol wt. (kDa) & Exons & Isoforms \\
\hline$\overline{\text { CaARF1 }}$ & LOC101492112 & XP_004485416 & $\mathrm{Ca} 1$ & 154,447 & 160,822 & + & 670 & 5.63 & 74.764 & 15 & 2 \\
\hline CaARF2 & LOC101513952 & XP_004485844 & $\mathrm{Ca} 1$ & $2,832,460$ & $2,837,619$ & - & 711 & 6.33 & 77.719 & 12 & 3 \\
\hline CaARF3 & LOC101501408 & XP_004485979 & Cal & $3,851,446$ & $3,857,899$ & - & 908 & 6.17 & 100.502 & 14 & 1 \\
\hline CaARF4 & LOC101509547 & XP_004487099 & Ca1 & $12,145,610$ & $12,149,407$ & + & 719 & 8.56 & 79.314 & 5 & 3 \\
\hline CaARF5 & LOC101492916 & XP_012571810 & Ca1 & $12,333,186$ & $12,338,793$ & + & 826 & 6.69 & 92.462 & 12 & 1 \\
\hline CaARF6 & LOC101498659 & XP_004488112 & Ca1 & $24,068,315$ & $24,076,322$ & - & 1120 & 6.07 & 125.619 & 14 & 3 \\
\hline CaARF7 & LOC101504978 & XP_004490754 & $\mathrm{Ca} 2$ & $28,016,288$ & $28,024,622$ & - & 833 & 5.89 & 92.432 & 14 & 1 \\
\hline CaARF8 & LOC101503141 & XP_004490828 & $\mathrm{Ca} 2$ & $29,095,001$ & $29,096,913$ & + & 504 & 6.63 & 55.295 & 2 & 1 \\
\hline CaARF9 & LOC101491204 & XP_012568938 & Ca3 & $14,022,096$ & $14,027,221$ & + & 671 & 5.89 & 75.11 & 13 & 1 \\
\hline CaARF10 & LOC101505543 & XP_004497510 & $\mathrm{Ca} 4$ & $20,116,751$ & $20,120,816$ & - & 692 & 7.05 & 77.313 & 4 & 1 \\
\hline CaARF11 & LOC101509304 & XP_012570835 & $\mathrm{Ca} 4$ & $48,380,932$ & $48,386,526$ & - & 917 & 5.55 & 102.376 & 15 & 1 \\
\hline CaARF12 & LOC101496441 & XP_012571326 & $\mathrm{Ca} 5$ & $27,748,317$ & $27,758,333$ & - & 853 & 5.95 & 94.344 & 16 & 2 \\
\hline CaARF13 & LOC101504083 & XP_004503553 & $\mathrm{Ca} 6$ & $4,214,355$ & $4,221,885$ & + & 1120 & 6.41 & 123.626 & 14 & 2 \\
\hline CaARF14 & LOC101498188 & XP_004503803 & $\mathrm{Ca} 6$ & $6,141,652$ & $6,146,690$ & - & 867 & 6.09 & 96.712 & 14 & 1 \\
\hline CaARF15 & LOC101500671 & XP_004504542 & $\mathrm{Ca} 6$ & $12,432,295$ & $12,439,057$ & + & 918 & 6.17 & 102.686 & 14 & 2 \\
\hline CaARF16 & LOC101493974 & XP_004505103 & $\mathrm{Ca} 6$ & $17,365,769$ & $17,371,522$ & - & 725 & 6.32 & 80.25 & 10 & 2 \\
\hline CaARF17 & LOC101505359 & XP_012572776 & $\mathrm{Ca} 6$ & $27,667,714$ & $27,674,248$ & - & 807 & 6.53 & 89.683 & 12 & 2 \\
\hline CaARF18 & LOC101492451 & XP_004506012 & $\mathrm{Ca} 6$ & $28,196,797$ & $28,201,150$ & - & 706 & 7.03 & 78.446 & 4 & 1 \\
\hline CaARF19 & LOC101515039 & XP_012572936 & Ca6 & $41,935,507$ & $41,941,343$ & + & 691 & 6.23 & 76.888 & 16 & 1 \\
\hline CaARF20 & LOC101489666 & XP_004508019 & $\mathrm{Ca} 7$ & $3,353,577$ & $3,360,886$ & - & 1125 & 6.53 & 126.203 & 15 & 7 \\
\hline CaARF21 & LOC101514738 & XP_004510646 & $\mathrm{Ca} 7$ & $34,898,868$ & $34,902,262$ & + & 612 & 7.61 & 69.328 & 4 & 2 \\
\hline CaARF22 & LOC101492136 & XP_004510662 & $\mathrm{Ca} 7$ & $35,086,399$ & $35,091,870$ & - & 679 & 6.38 & 75.972 & 15 & 3 \\
\hline CaARF23 & LOC101505502 & XP_004511136 & $\mathrm{Ca} 7$ & $44,457,833$ & $44,469,177$ & + & 444 & 5.72 & 50.687 & 12 & 1 \\
\hline CaARF24 & LOC101514889 & XP_012567350 & * & * & * & * & 598 & 6.17 & 65.776 & 4 & 1 \\
\hline
\end{tabular}




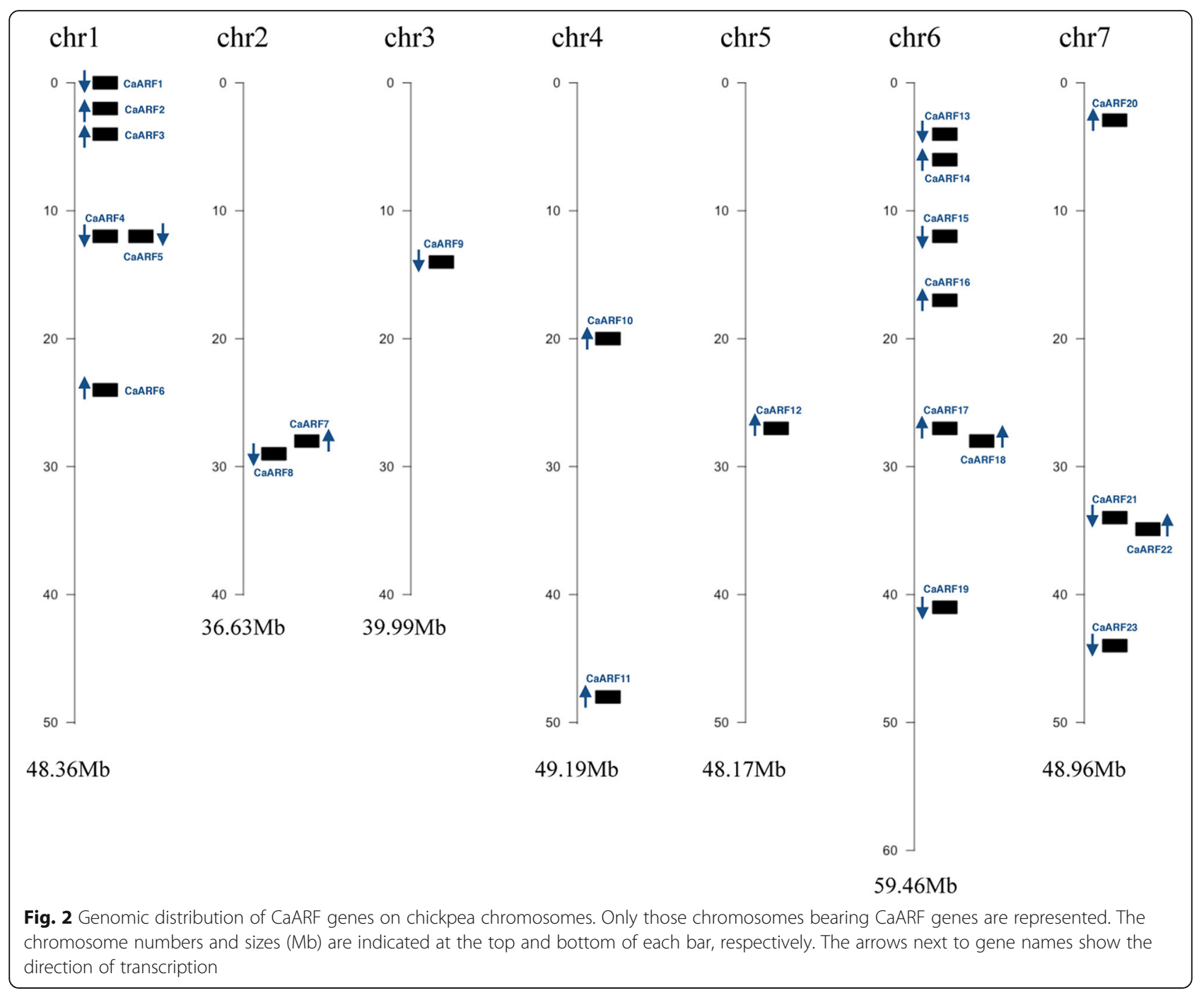

In addition, further investigation showed that two genes (CAARF15 and CaARF16) were located in the vicinity of two QTL clusters (cluster 7 and 8) in chromosome 6 (Linkage Group LG6) associated to drought component traits in chickpea [55]. Those QTLs were located in a consensus map derived from two recombinant inbred line populations. Cluster 7 , is pointed by the microsatellite marker NCPGR200 (physical position 17,478,000-17,478,700) and comprised some genomic regions associated to days to flowering, days to maturity and harvest index (QR4df02, QR4dm03 and QR4hi02 respectively). Cluster 8, with indicative marker TA106 (12,589,04012,589,266) included QR3rsa02, QR3pht01 and QR3dm03 for root surface area, plant height and days to maturity respectively. Further studies on these genomic regions will offer insight about the potential role of these ARF for drought tolerance and their value for chickpea breeding.

\section{Analysis of amino acid composition and classification of} CaARF

Next, we aligned and analyzed the amino acid sequences of the CaARF. The domain positions in the 24 CaARF proteins are shown in Additional file 4: Table S1. Sequence analysis of the CaARF proteins, Pfam protein motif- and CDD analyses showed that most of them (18 ARF proteins) harbour the typical ARF structure comprising (1) a highly conserved DNA-binding domain (DBD) in the $\mathrm{N}$-terminal region made of a plant specific B3-type subdomain and an AUX_RESP subdomain, (2) a variable middle region (MR) that functions as an activation or repression domain, and (3) a carboxyterminal dimerization (CTD) domain similar to that found in Aux/IAA proteins. All proteins with length < 670 aa showed only the DBD domain (B3 and AUX RESP), whereas all proteins $>725$ aa contained the DBD and CTD domains (B3, AUX_RESP and Aux/IAA; Additional file 3: Figure S3). In the canonical ARF 
structure model, the CTD is made of two highly conserved subdomains (III and IV). Fifteen of those 18 proteins fit into that model, whereas three ARF have a truncated CTD since only subdomain III is present (CaARF4, 10, 18). Six proteins lack domains III and IV.

Then, we analyzed the amino acid composition of the Middle Regions (MRs). Seven proteins harbour a glutamine $(\mathrm{Q})$, serine $(\mathrm{S})$ and leucine $(\mathrm{L})$-rich middle region implying that these proteins are likely transcriptional activators since glutamine enrichment seems to be a distinctive feature of ARF activators in all plant lineages $[4,6]$. The other eleven CaARF proteins containing a CTD domain may function as repressors based on their MRs enriched in serine, proline, glycine and leucine (SPGL). Six CaARF proteins lacking the CTD domain may also be repressors based on their MR amino acid enrichment (Additional file 5: Figure S4 and Additional file 6: Table S2). The activator/repressor ratio among CaARF is 0.41, which is higher than that in Medicago (0.26) but lower than Arabidopsis (0.59).

It should be noted that the classification of the ARF family into either activators or repressors is based merely on the enrichment of the specific amino acids but it is unclear what mechanisms underlie activation and repression. Therefore, the activator/repressor categorization should be exercised with caution [56].

\section{Comparative phylogenetic analysis of the ARF family}

To study the phylogenetic relationships between the members of CaARF gene family and to explore the phylogenetic relationships of ARF genes among different species, we used the model plants Arabidopsis and Medicago. An unrooted tree was constructed from an alignment of 23 AtARF, 24 MtARF and 24 CaARF proteins. The phylogenetic distribution of the 71 ARF revealed that all ARF sequences fall into two major groups (I and II) with well-supported bootstrap values (Fig. 3). Groups II and I are further subdivided into four and two subgroups, respectively. The distribution of each class of CaARF was significantly irregular as the chickpea proteins located on the same chromosome belonged to different groups and subclasses. It has been hypothesized that ARF on the same chromosome may have complementary functions [54]. The group I is the most numerous and contains 18 CaARF, 20 AtARF and 11 MtARF proteins. We labeled as sister pairs those proteins clustered together based on high bootstrap values (>65\%). Related to sister pairs involving chickpea, the group I structures 10 sister pairs (nine pairs of CaARF-MtARF and one pair of CaARF-AtARF). Interestingly, we did not find any sister pair between two chickpea ARF proteins. Chickpea diverged from M. truncatula $\sim 10-20$ million years ago (Mya; [19]). Lack of chickpea sister pairs suggests that recent duplications (after chickpea and Medicago separated) have played a very limited role, if any, in the expansion of the ARF chickpea family. All seven potential activator CaARF proteins containing the canonical structure DBD-MR-CTD clustered in subclade Ia. Subclass Id is a lineage-specific clade found in the Arabidopsis ARF family. It contains the seven tandem duplicated genes (encoding proteins AtARF12 to ARF15 and AtARF20 to ARF22; [57]) with no homology to any chickpea ARF sequence. Other plant species, such as rice, maize, tomato and grape or Eucalyptus, also lack homologs to that subclass [52, 54, 58-60], implying that these AtARF were derived through a long-term evolution for Arabidopsis-specific functions [21]. Between class Ic and Id, an isolated clade clusters CaARF23, with no obvious Arabidopsis ortholog. We found that CaARF23 shares $43 \%$ identity with the Eucalyptus grandis ARF24 at the amino acid level. The EgrARF24 protein has also been clustered in an isolated clade without any Arabidopsis ortholog [60]. That clade is absent from the herbaceous annual plants (Arabidopsis, tomato and rice) but present in Eucalyptus and other woody perennials, so the authors stated that it might be a woodypreferential clade [60]. However, the same authors identified two members of the legume family (G. max and $P$. vulgaris) as members of that clade. We found that CaARF23 shared high degree of similarity with those legume proteins (Additional file 7: Figure S5). Therefore, rather than a woody-preferential clade, it is more likely that these are orthologs of an ancestral gene lost in Arabidopsis but present in woody perennials as well as some legume species. Finally, group II contains six chickpea ARF members. Three sister pairs (all of them pairs of CaARF-MtARF) were confirmed based on bootstrap values above 90\%. Group II also contains the three Arabidopsis members (AtARF10, ARF16 and ARF17) that are the most divergent compared with those encoded by the other class [57]. The subclass IIa is made mostly of Medicago ARF sequences (10 MtARF, 2 CaARF, 1AtARF) indicating a diverging trend in the evolution of ARF family members across different plant species.

The exon-intron organization of a gene family can provide additional evidences to support the evolutionary relationship among all members. Gene structure tends to remain the same within genes present in the same clade, whereas dissimilarity may be found within clades. The coding sequences of CaARF clustered in group II showed a strong conservation of their gene structure and had an average exon number noticeably lower than those CaARF clustered in group I (3.8 vs. $13.7 ; \mathrm{t}$ test, $P<1 \mathrm{e}-11)$. The phenomenon of different exon number has been observed in other species, such as Arabidopsis, rice and $B$. rapa $[30,52,57,61]$. 


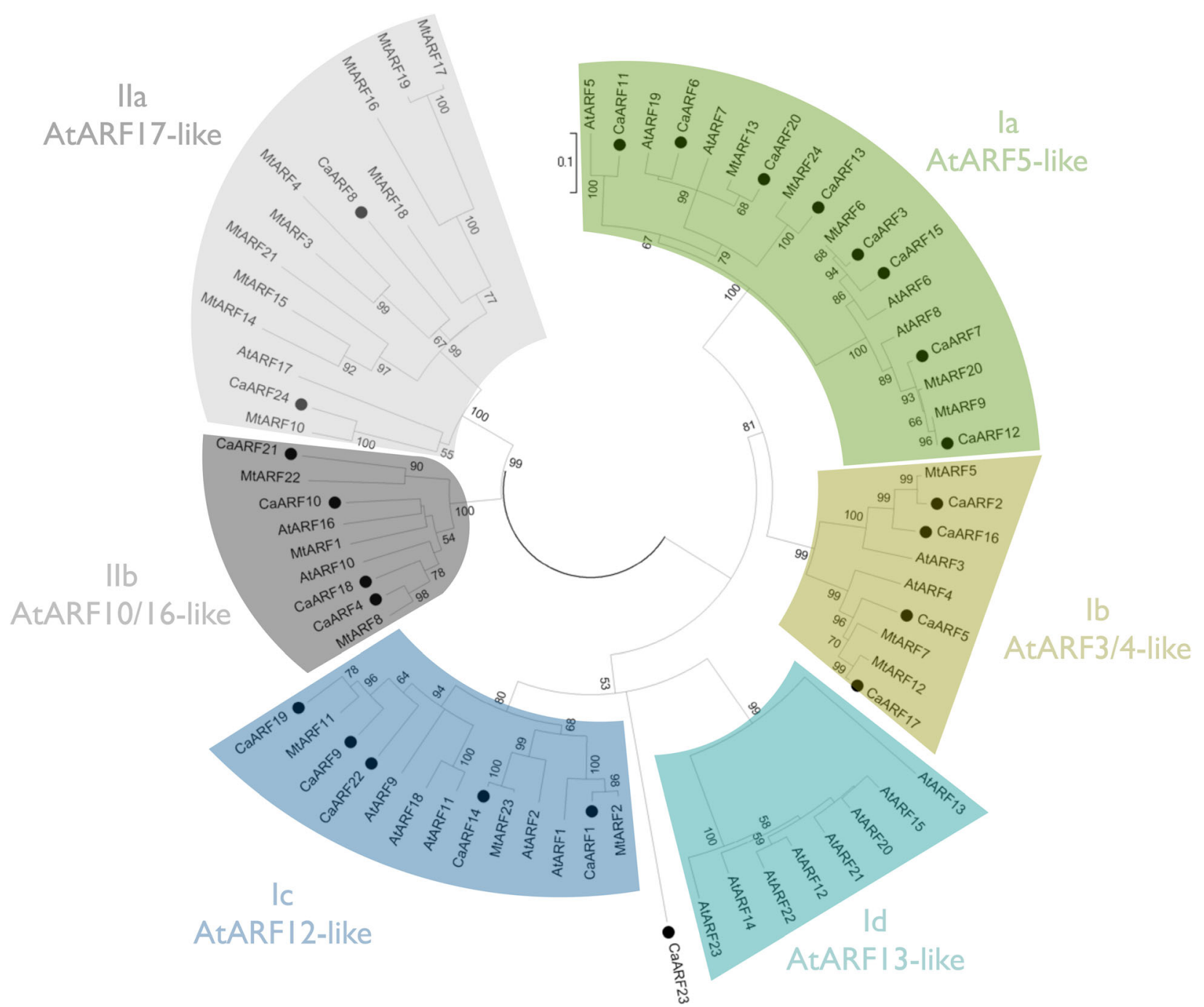

Fig. 3 Analysis of phylogenetic relationships of ARF in C. arietinum, M. truncatula and A. thaliana. Twenty-four CaARF proteins, 24 MtARF and 23 AtARF were classified into six subclasses. Scale bar 0.1 denotes the number of amino acid substitutions per site. The percentage of replicate trees in which the associated taxa clustered together in the bootstrap test (1000 replicates) is shown next to the branches. CaARF are marked with solid black circles

\section{Diversification of CaARF}

We found two major molecular mechanisms underlying the diversification of CaARF proteins, namely diversification by expansion (quantitative diversification) and structural diversification (qualitative diversification). It is important to note that both categories are merely descriptive as they are interconnected and play substantial contribution to the diversity of ARF proteins. For example, genome duplication events are important not only for the numeric expansion of a gene family but also for genomic rearrangements and therefore, for diversification of gene function [6].

\section{Diversification by expansion}

Duplicated genes represent the source of genetic materials for studying evolution and diversification [62]. We performed duplication analysis using the PGDD software to find the potential relationships between putative paralog pairs of ARF genes and tandem/segmental duplications. ARF genes in tandem clusters have been detected in Medicago, Arabidopsis, and peach [20, 57, 63].

Chromosomes with more than one ARF gene are candidates to have undergone local gene duplications. We found two genes on chromosome 1 (ARF4-5) and two genes on chromosome 7 (ARF21-22) that met the criteria to form a cluster as it has been described in the Materials and Methods section. These two pairs are separated by $<190 \mathrm{~kb}$ in each case. However, both pairs show relative low values of similarity. CaARF4 and CaARF 5 show $40 \%$ identity at $62 \%$ query coverage (Evalue $=9 \mathrm{e}-81$ ), while $C a A R F 21$ and $C a A R F 22,34 \%$ identity 
at $64 \%$ coverage (Evalue $=7 \mathrm{e}-65$ ). These clusters might have been produced by ancient tandem duplication events. On the other hand, it appears that a number of genes (12 genes, 50\%) were segmentally duplicated (Additional file 8: Figure S6). The rate of synonymous substitution per synonymous site (Ks) was used as a proxy for time and the segmental duplications of the CaARF genes were assumed to originate from 48 Mya (million years ago, Ks $=0.59)$ to 134 Mya $(\mathrm{Ks}=1.64)$. Most of the segmental CaARF duplications seem to have occurred 50-60 Mya. Interestingly, the duplication pattern observed in the CaARF family coincides with the Ks rates found over all the systemic blocks in the chickpea genome that indicates a divergence time of 58 Mya ago [19]. This observation corresponds with the occurrence of whole-genome duplication (WGD) event that occurred at the base of the Papilionoideae (58-60 Mya ago; [64]. Therefore, most of the CaARF duplications originated during the WGD that occurred prior to the speciation of legumes. In order to detect the mode of selection, we evaluated the ratio of nonsynonymous to synonymous nucleotide substitutions $(\mathrm{Ka} / \mathrm{Ks})$ among paralogs [65]. Generally, a Ka/Ks ratio $>1$ indicates positive selection; a pair of sequences will have a ratio $<1$ if one sequence has been under purifying selection, but the other has been drifting neutrally; and a ratio $=1$ indicates that both sequences are under neutral evolution [35]. As shown in Additional file 9: Table S3, the average $\mathrm{Ka} / \mathrm{Ks}$ value of the CaARF gene pairs was 0 . 19. Most $\mathrm{Ka} / \mathrm{Ks}$ ratios ranged from 0.11 to 0.27 and none of them was $>1$.

These results suggest that essentially, segmental duplications, but not tandem duplications, have contributed to the expansion of the ARF gene family in chickpea. Moreover, the duplicated pair genes have evolved mainly under the influence of purifying selection pressure with no functional divergence after segmental duplications.

\section{Structural diversification}

Increasing the number of genes within a group through duplication events contribute to the expansion of that group. Another mechanism underlying diversity is domain rearrangements, which involves variations in domain organization. In case of ARF proteins, the alternative domain organizations are illustrated by losses of domains III and/or IV. Numerous and independent losses of domains III and IV seems to have occurred during land plant evolution [6]. However, in spite of these truncations, the proteins are all functional. As said before, six CaARF proteins lack domains III and IV, whereas three ARF have a partial truncated CTD since only subdomain III is present (CaARF4, 10, 18). Therefore, the percentage of CTD-truncated CaARF (37. $5 \%$ is more than twice as much as that identified in
Arabidopsis (17.39\%) but lower than in Medicago (54\%). This may suggest that $C$. arietinum shows a tight auxindependent transcriptional regulation, at least compared to Medicago. Truncated ARF lack domains of interaction with Aux/IAAs, a sequestration mechanism that is released in the presence of auxin. The truncated ARF are predicted to be unable to interact with Aux/IAA, and hence, they should consequently be insensitive to auxin [66]. Therefore the presence of a large number of CTD-truncated ARF in Medicago has been understood as an auxin-independent transcriptional regulation [63]. Nevertheless, the true functional significance of truncations is not well understood and this point remains still open. ARF activators may fit well under the insensitiveto-auxin scenario. However, this hypothesis seems unlikely to be relevant for ARF repressors, which have limited interactions with Aux/IAA proteins [5, 11]. For that reason, it has been pointed out that loss of domains III and IV could also have consequences on the interaction of ARF with other transcription factors [6].

Concerning the protein structure, alternative splicing represents an additional mechanism underlying diversity in ARF proteins. Extensive gene duplication and alternative splicing have generally been viewed as opposite trends in gene family evolution $[67,68]$. However, ARF proteins represent a clear example in which both processes play a significant role in functional diversification [6]. Computational survey of the alternative transcripts predicted in the $C$. arietinum genome revealed that at least half of the gene family members display alternative splicing. Seven ARF genes have evidence of two alternative variants, four genes of three variants, and one gene (CaARF20 on Chr7) shows up to seven different variants. Thus, the number of possible alternative transcripts in the chickpea genome is 45 . It would be of interest to further characterize the functional roles of different chickpea isoforms.

Although both, genomic truncations and alternative splicing are diversification mechanisms occurring in a non-preferential chromosome location, we found that they had a differential evolutionary significance. Genomic truncations are mainly restricted to clade II and isolated nodes of subclades Ib and Ic. Alternative splicing, on the contrary, is of widespread occurrence with distribution in branches of subclades Ia, Ib, Ic, and IIb (Additional file 10: Figure S7).

\section{Gene expression patterns of CaARF}

In a first attempt to gain an insight into the putative function of ARF genes in chickpea, we analyzed their expression profiles in different tissues using EST available datasets [69]. Considering the stringent criterion described in the Material and Methods section, nine ARF genes had expression data support (14 ESTs). One ARF 
(CaARF23) hit 3 ESTs, whereas three ARF (CaARF1, 14, 15) were supported at least by 2 ESTs (Additional file 11: Table S4). Regarding the plant tissues, roots and leaves were the most common hit, although 1 EST from shoot and 1 from embryo were also found. Concerning the experimental conditions, the EST libraries were generated from drought and salinity conditions (11 ESTs in total), during the interaction with the necrotrophic fungus $A$. rabiei, (2 ESTs) and during the embryo development (1 EST). The EST data mining suggests a variety of plant processes and responses to the environment played by ARF. Then, we aimed at measuring the expression levels of the ARF genes in 22 samples representing mainly the tissues and stress conditions showed by the ESTs. The transcript levels for the 24 ARF genes were determined by RT-qPCR. Melting curves showed non-specific PCR product amplification for the $A R F 8$ primer pair, so that gene was not further considered. We performed singular value decomposition analysis to evaluate the contribution of the stresses and treatments to the variance within the transcript dataset. The largest variance indicated by the first dimension accounts for $35.6 \%$ of the total variance in the dataset, while the second dimension accounts for $21.6 \%$ of the total variance. This suggests that the dataset contains a moderately high explanatory signal (explaining 57.2\%). A multidimensional scaling plot showed that the separation of the samples along the first and second dimension is mainly driven by the tissue (Fig. 4a). Our data support the findings in the model legume $M$. truncatula, whose ARF show complementary tissue-specific auxin responsiveness, with one ARF group induced in shoots and down-regulated in roots, while another group shows the opposite effect [63]. Consistently, when we compared leaves and roots of well-

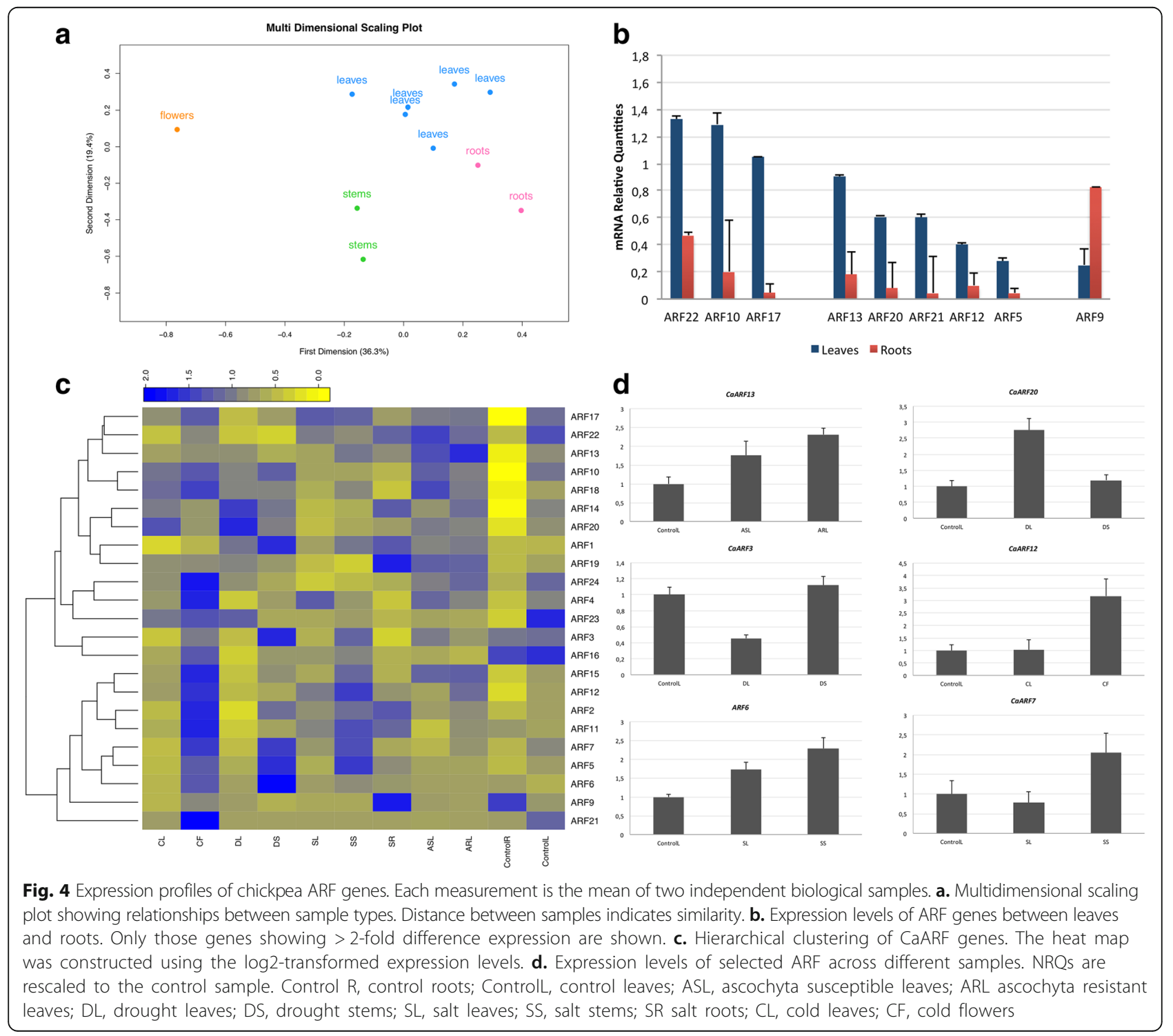


developed plants, we identified a set of ARF expressed at higher levels in leaves than in roots (differences $>2$-fold change compared to the reference genes). A second group showed low expression in both, leaves and roots, but the genes were expressed with higher levels in leaves. Finally, CaARF9 was the only gene that showed higher expression in root tissues vs leaves (Fig. 4b).

The hierarchical clustering of the $\log 2$ fold-change values shows that the ARF genes clustered into two main expression groups. A heat map representation (Fig. 4c) indicated that the first group is the largest with 14 genes and contains three predicted activators (ARF3, 13, and 20 ). The group is mostly made of genes clustered into clades Ic and IIb (Fig. 3). All genes encoding proteins with a truncated structure but one (ARF2), fell into this group. The second group is smaller and contains nine members including the other four activators (ARF6, 7, 12, and 15). Most of the genes clustered into clades Ia and Ib fell into this group, which is made of genes encoding proteins with the canonical structure, with the exception of ARF2. The heat map representation also indicated that different members display preferences to particular samples. Concerning the first group genes ARF17, 22, 13, 10, 18, 14 and 20 showed extremely low values in well-developed roots but we measured higher levels in roots from plants exposed to salt (ARF14, 22). Some genes in this group also showed increased levels related to controls in leaves inoculated with aschochyta blight (ARF22, 13, 10, and 18) or leaves from plants under drought (ARF20). Finally, the expression of the predicted activator $A R F 3$ peaked in stems from drought plants but showed low expression leaves under salinity conditions (Fig. 4d). Genes clustered in the second group are preferentially expressed in flowers and stems. This group is virtually absent from leaves. We only detected high expression levels for ARF12 and 15 when leaves were from samples infected with ascochyta blight.

\section{Cis-regulatory elements in promoters of CaARF}

An important question is how ARF can regulate genes in the context of chromatin. Recently, a chromatin switch mechanism has been proposed to direct ARF-dependent gene activation [70]. Aux/IAA proteins compete with SWI-SNF (SWITCH/SUCROSE NONFERMENTING) recruitment, and thus the Aux/IAA degradation allows chromatin remodeling. This mechanism makes the chromatin region more accessible for other transcription factors [71]. In order to give some insight into the dynamic regulation of ARF we analyzed the occurrence and distribution of cis-regulatory elements (CREs) over their promoter sequences. The expected frequency of each CRE was calculated using the average $\mathrm{G}+\mathrm{C}$ content of $27.8 \%$ observed in the chickpea dataset (range 26.8 to 32.2). We screened the proximal and distal regions of promoters (up to $1500 \mathrm{bp}$ upstream of the transcriptional start site, TSS) to identify candidate cis-elements that might contribute to the fine regulation of gene expression at the transcriptional level. We were able to retrieve the promoter genomic sequences from the 23 ARF genes that were mapped onto chromosomes. To analyze the promoter regions, first the $1.5 \mathrm{~kb}$ of genomic DNA sequences upstream of TSS were used to query the GenBank database (nr) by BLASTx. The results confirmed that these surveyed sequences are not coding sequences. Next, we estimated the number, abundance and position of some CREs that had been associated previously to biotic, abiotic and auxin-dependent responses in the literature. Further statistical analysis indicated that the ARF gene promoters showed an enriched content of the CREs dataset (Table 2). The element CTCTT, which is involved in symbiosis, appeared a total of 77 times in the whole set of 23 promoters (average 3.35 elements/ promoter). Motif CTCTT might have an evolutionary conserved function in controlling plant gene expression during the interaction with microbes [48]. The abundance of the element CTCTT in all the chickpea promoters indicates that this element is important for regulation of ARF genes and provides further evidence that auxin signalling plays a central role during plantmicrobe interactions [72]. Most of the sequences also contained the frequent elements GT1GMSCAM4 and RAV1AAT (22 promoters each). On the other hand, the AGCBOXNPGLB element was present in only one promoter $(n=1)$. However, this CRE was also clearly overrepresented in our query set. Finally, we tested whether any given CRE is more common in certain promoter regions compared to background. For this, the promoter sequences were divided into $100 \mathrm{bp}$ nucleotide fragments and the content of the 5 CREs most enriched in our dataset was calculated. An increase in density of the fragments was identified at a distance of $\sim-600$ to $400 \mathrm{bp}$ from the TSS, whereas the control set produced CREs with a uniform distribution in the promoter region (Additional file 12: Figure S8). This result indicates that the region of high distribution density $(-600$ to -400$)$ should be considered in the analysis of the auxin responsive regions.

\section{Conclusions}

In this study we have analyzed the chickpea genome to identify and characterize the ARF gene family by using a broad range of bioinformatic tools. The ARF proteins from $M$. truncatula were retrieved using the Phytozome database. We used NCBI BLAST searches for query of nucleotide and amino acid sequences in the CDC Frontier genome assembly. The HMM profiles were determined through Pfam and CDD databases. The pI was obtained on the ExPASy proteomics server database. 
Table 2 Cis-regulatory elements in chickpea ARF promoters

\begin{tabular}{|c|c|c|c|c|c|c|c|c|}
\hline CRE & Motif & Query $^{a}$ & $\begin{array}{l}\text { Promoters } \\
\text { Observed }\end{array}$ & $\begin{array}{l}\text { Total Occurrences } \\
\text { Observed }^{b}\end{array}$ & $\begin{array}{l}\text { Avg. Number Occurrs. } \\
\text { per promoter }\end{array}$ & $\begin{array}{l}\text { Total Occurrs. } \\
\text { Expected }^{c}\end{array}$ & $\begin{array}{l}\text { Enrichment } \\
\text { Factor }^{d}\end{array}$ & $P$-value \\
\hline AGCBOXNPGLB & AGCCGCC & 23 & 1 & 1 & 1.00 & 0.0575 & 17.39 & 0.0041 \\
\hline RAV1AAT & CAACA & 23 & 22 & 93 & 4.23 & 31.02 & 3.00 & 0.0337 \\
\hline GT1GMSCAM4 & GAAAAA & 23 & 22 & 72 & 3.27 & 28.98 & 2.48 & 0.0327 \\
\hline Motif CTCTT & CTCTT & 23 & 23 & 77 & 3.35 & 1.33 & 2.43 & 0.0325 \\
\hline SURE2STPAT21 & AATACAAAA & 23 & 3 & 3 & 1.00 & 1.58 & 1.90 & 0.0194 \\
\hline AuxRe1 & TGTCTC & 23 & 6 & 8 & 1.33 & 4.77 & 1.68 & 0.0293 \\
\hline WBBOXPCWRKY1 & TTTGACY & 23 & 7 & 8 & 1.14 & 1.37 & 1.41 & \\
\hline MYCATERD22 & CACATG & 23 & 6 & 7 & 1.17 & 4.53 & 1.54 & \\
\hline MYCATERD1 & CATGTG & 23 & 7 & 7 & 1.00 & 4.67 & 1.50 & \\
\hline ABRE & ACGTGTC & 23 & 1 & 1 & 1.00 & 0.69 & 1.45 & \\
\hline
\end{tabular}

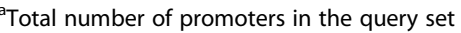

${ }^{\mathrm{b}}$ Total number of motifs in the query set

'Total number of motifs expected to occur by chance/1.5 kb promoter based on nucleotide frequency in 23 promoter sequences

${ }^{\mathrm{d}}$ Number of motifs observed divided by the number of motifs expected to occur by chance

eProbabilities based on 2000 Monte Carlo simulations

Chromosomal locations, locus ID, aa lengths, molecular weights and number of exons were retreived from the NCBI using custom $\mathrm{R}$ scripts. Multiple sequence alignment and phylogenetic tree construction were performed using MUSCLE and MEGA, respectively. Exon-intron distributions were analyzed using GSDS server. Gene duplications were determined using the PGDD database and the orthologous relationships were visualized using Circoletto. Amino acid content of the MR domain was calculated using the 'Biostrings' Bioconductor package. The putative cis-acting regulatory elements in the promoters were analyzed using custom Python scripts. In silico expression data were obtained via the NCBI EST database. The physical map of chromosomal location was generated using the 'IRanges' Bioconductor package. The heat map for expression profile was constructed using the 'Stats' R package.

Our data suggest that segmental duplications have contributed to the expansion of the ARF gene family in chickpea. The duplicated pairs have evolved mainly under the influence of purifying selection pressure. Genomic truncation and alternative splicing are also important mechanisms for the diversity of the ARF family. Although genomic truncations are restricted to specific clades, alternative splicing shows a widespread distribution. Expression profiles show a close relationship between tissue and expression patterns. Most of the genes from the same phylogenetic class also clustered in one expression branch. This may indicate that ARF genes from the same class perform similar physiological function in chickpea. The expression results give support for various functional roles of ARF genes in a wider range of developmental processes and stresses. Our study also provides a foundation for further comparative genomic analyses and a framework to trace the dynamic evolution of ARF genes on a large time-scale within the Papilionoideae family.

\section{Additional files}

Additional file 1: Figure S1. geNorm ranking of 4 reference genes from chickpea samples. The expression stability value $(M)$ is shown as bar plot. Vertical numbers at the top indicate the CV values of the reference genes involved in the normalization. The best pair of references (highly stable expression with $M$ values $<1$ and $C V<0.5$ ) is represented as black bars. (PDF $190 \mathrm{~kb}$ )

Additional file 2: Figure S2. ARF protein identity between chickpea, Arabidopsis and Medicago. (PDF $68 \mathrm{~kb}$ )

Additional file 3: Figure S3. ARF gene family in chickpea. a. Distribution of CaARF on chromosomes based on protein length. $\mathbf{b}$. HMM profiles of CaARF based on protein length. (PDF $43 \mathrm{~kb}$ )

Additional file 4: Table S1. Domain positions in 24 CaARF proteins. (PDF $48 \mathrm{~kb}$ )

Additional file 5: Figure S4. Protein structure of CaARF family. DBD, DNA-binding domain; MR, middle region; CTD, C-terminal dimerization domain; $A D$, activation domain (orange color); $R D$, repression domain (green color); Q, glutamine; S, serine; L, leucine; P, proline; G, glycine. (PDF $32 \mathrm{~kb}$ )

Additional file 6: Table S2. Data of amino acid content in MR domain of CaARF. (PDF $76 \mathrm{~kb}$ )

Additional file 7: Figure S5. Phylogenetic relationships between the orthologs of CaARF23 in other species. The phylogenetic tree was constructed using the Arabidopsis AtARF2 as an outgroup. The species shown in the figure are Gossypium raimondii (2), Theobroma cacao (1), Citrus clementine (1), Citrus sinensis (1), Populus trichocarpa (2), Vitis vinifera (1), Fragaria vesca (1), Prunus persica (1), Malus domestica (2), Eucalyptus grandis (1), Carica papaya (1), Phaseolus vulgaris (1), Glycine max (2), and Aquilegia coerulea (1). (PDF $55 \mathrm{~kb}$ ) 
Additional file 8: Figure S6. Similarity of CaARF genes. Red color shows highest similarity (> $80 \%$ identity) followed by orange $(70-80 \%)$ and green $(60-70 \%)$ colors. (PDF $12 \mathrm{~kb})$

Additional file 9: Table S3. Duplicated gene pairs of CaARF genes with $\mathrm{Ka} / \mathrm{Ks}$ values and time of duplication. (PDF $52 \mathrm{~kb}$ )

Additional file 10: Figure S7. Gene structure and transcripts analyses of ARF members in chickpea. The figure shows members with genomic truncation (losses of domains III and/or IV), and alternative variants. (PDF $111 \mathrm{~kb}$ )

Additional file 11: Table S4. Tissue distribution profile of chickpea ARF genes according to the number of expressed sequence tags (ESTs) present in NCBI's EST Database. (PNG 886 kb)

Additional file 12: Figure S8. Distribution de CRES. a. Simulated data set. b. Actual data set. (PDF $47 \mathrm{~kb}$ )

\section{Abbreviations}

ARF: Auxin response factor proteins; Aux/IAA: Auxin/Indole-3-Acetic Acid; CDD: Conserved Domain Database; CREs: Cis-regulatory elements; CTD: carboy-terminal dimerization domain; DBD: DNA-binding domain; E: Real-time GPCR amplification efficiency; HMM: Hidden Markov model; Ka: Nonsynonymous substitutions per nonsynonymous site; Ks: Synonymous substitution per synonymous site; MR: Middle region; Mya: Million years ago; pl: Isoelectric point; QTL: Quantitative trait loci; RT-qPCR: Reverse transcription quantitative real-time PCR; TSS: Transcriptional start site; WGD: Wholegenome duplication.

\section{Funding}

INIA RTA2017-00041-00-00, project co-financed by the European Union through the ERDF 2014-2020 "Programa Operativo de Crecimiento Inteligente" and Contract 77863. The funding body had no role in the design of the study and collection, analysis and interpretation of data and in writing the manuscript.

\section{Availability of data and materials}

All data generated or analyzed during this study are included in this article and its supplementary information files.

\section{Authors' contributions}

JVD conceived the study and performed bioinformatics analyses. JG supervised and revised the outgoing data. TM performed the mapping analysis for ARF genes to molecular markers for agronomical traits. All authors contributed to the critical writing with JVD taking the lead on the writing. All authors read and approved the manuscript.

\section{Ethics approval and consent to participate}

All plant materials used in this study come from our chickpea breeding program at Department of Genetics - ETSIAM, University of Córdoba. Inoculations with isolate of Ascochyta rabiei were performed under controlled conditions, using standard protocols, and in accordance with legislation of University of Córdoba.

\section{Competing interests}

The authors declare that they have no competing interests.

\section{Publisher's Note}

Springer Nature remains neutral with regard to jurisdictional claims in published maps and institutional affiliations.

\section{Received: 27 December 2017 Accepted: 18 April 2018}

Published online: 27 April 2018

\section{References}

1. Chapman EJ, Estelle M. Mechanism of auxin-regulated gene expression in plants. Annu Rev Genet. 2009;43:265-85.

2. Guilfoyle TJ, Hagen G. Auxin response factors. Curr Opin Plant Biol. 2007;10: 453-60

3. Ulmasov T, Hagen G, Guilfoyle TJ. ARF1, a transcription factor that binds to auxin response elements. Science (New York, NY). 1997;276:1865-8.
4. Ulmasov T, Hagen G, Guilfoyle TJ. Activation and repression of transcription by auxin-response factors. Proc Natl Acad Sci U S A. 1999:96:5844-9.

5. Vernoux T, Brunoud G, Farcot E, Morin V, Van den Daele H, Legrand J, et al. The auxin signalling network translates dynamic input into robust patterning at the shoot apex. Mol Syst Biol. 2011;7:508.

6. Finet C, Berne-Dedieu A, Scutt CP, Marlétaz F. Evolution of the ARF gene family in land plants: old domains, new tricks. Mol Biol Evol. 2013; 30:45-56.

7. Weijers $D$, Benkova $E$, Jäger KE, Schlereth $A$, Hamann $T$, Kientz M, et al. Developmental specificity of auxin response by pairs of ARF and aux/IAA transcriptional regulators. EMBO J. 2005;24:1874-85.

8. De Smet I, Lau S, Voss U, Vanneste S, Benjamins R, Rademacher EH, et al. Bimodular auxin response controls organogenesis in Arabidopsis. Proc Natl Acad Sci U S A. 2010;107:2705-10.

9. Tiwari SB, Hagen $G$, Guilfoyle $T$. The roles of auxin response factor domains in auxin-responsive transcription. Plant Cell. 2003;15:533-43.

10. Ulmasov T, Hagen G, Guilfoyle TJ. Dimerization and DNA binding of auxin response factors. Plant J. 1999;19:309-19.

11. Piya S, Shrestha SK, Binder B, Stewart CN, Hewezi T. Protein-protein interaction and gene co-expression maps of ARFs and aux/IAAs in Arabidopsis. Front Plant Sci. 2014:5:744.

12. Kim J, Harter K, Theologis A. Protein-protein interactions among the aux/IAA proteins. Proc Natl Acad Sci U S A. 1997:94:11786-91.

13. Bolger ME, Weisshaar B, Scholz U, Stein N, Usadel B, Mayer KF. Plant genome sequencing - applications for crop improvement. Curr Opin Biotechnol. 2014;26:31-7.

14. FAOSTAT. FAOSTAT Crop Statistics. 2017. Latest update: May 17, 2017. Latest update: May 17, 2017. URL http://www.fao.org/faostat. Accessed Nov 2017.

15. Li H, Rodda M, Gnanasambandam A, Aftab M, Redden R, Hobson K, et al. Breeding for biotic stress resistance in chickpea: progress and prospects. Euphytica. 2015;204:257-88.

16. Millan T, Madrid E, Cubero Jl, Amri M, Castro P, Chickpea RJ. Grain Legumes. New York: Springer New York; 2015. p. 85-109.

17. Varshney RK. Exciting journey of 10 years from genomes to fields and markets: some success stories of genomics-assisted breeding in chickpea, pigeonpea and groundnut. Plant Sci. 2016:242:98-107.

18. Jain M, Misra G, Patel RK, Priya P, Jhanwar S, Khan AW, et al. A draft genome sequence of the pulse crop chickpea (Cicer arietinum L.). Plant J. 2013;74:715-29.

19. Varshney RK, Song C, Saxena RK, Azam S, Yu S, Sharpe AG, et al. Draft genome sequence of chickpea (Cicer arietinum) provides a resource for trait improvement. Nat Biotechnol. 2013;31:240-6.

20. Li H, Ran K, Sun Q. Genome-wide identification and expression analysis of peach auxin response factor gene families. J Plant Biochem Biotechnol. 2016:25:349-57.

21. Van Ha C, Le DT, Nishiyama R, Watanabe Y, Sulieman S, Tran UT, et al. The auxin response factor transcription factor family in soybean: genome-wide identification and expression analyses during development and water stress. DNA Res. 2013:20:511-24.

22. Altschul SF, Gish W, Miller W, Myers EW, Lipman DJ. Basic local alignment search tool. J Mol Biol. 1990;215:403-10.

23. Marchler-Bauer A, Bo Y, Han L, He J, Lanczycki CJ, Lu S, et al. CDD/SPARCLE: functional classification of proteins via subfamily domain architectures. Nucleic Acids Res. 2017:45:D200-3.

24. Edgar RC. BMC bioinformatics. BMC Bioinformatics. 2004:5:113-9.

25. Pages $H$, Aboyoun P, Gentleman R, DebRoy S. Biostrings: string objects representing biological sequences, and matching algorithms. $\mathrm{R}$ package version 2. 2016;42:1

26. Saitou N, Nei M. The neighbor-joining method: a new method for reconstructing phylogenetic trees. Mol Biol Evol. 1987;4:406-25.

27. Felsenstein J. Confidence limits on phylogenies: an approach using the bootstrap. Evolution. 1985:39:783.

28. Zuckerkandl E, Pauling L. Evolutionary divergence and convergence in proteins. In: Bryson V, Vogel HJ, editors. Evolving genes and proteins a symposium held at the Institute of Microbiology of Rutgers: the state university with support from the National Science Foundation. New York: Elsevier; 1965. p. 97-166

29. Tamura K, Peterson D, Peterson N, Stecher G, Nei M, Kumar S. MEGA5: molecular evolutionary genetics analysis using maximum likelihood, evolutionary distance, and maximum parsimony methods. Mol Biol Evol. 2011;28:2731-9. 
30. Hu B, Jin J, Guo A-Y, Zhang H, Luo J, Gao G. GSDS 2.0: an upgraded gene feature visualization server. Bioinformatics (Oxford, England). 2015;31:1296-7.

31. Lee T-H, Tang H, Wang X, Paterson AH. PGDD: a database of gene and genome duplication in plants. Nucleic Acids Res. 2013;41:D1152-8.

32. Darzentas N. Circoletto: visualizing sequence similarity with Circos. Bioinformatics (Oxford, England). 2010;26:2620-1.

33. Ameline-Torregrosa C, Wang B-B, O'Bleness MS, Deshpande S, Zhu H, Roe B, et al. Identification and characterization of nucleotide-binding site-leucinerich repeat genes in the model plant Medicago truncatula. Plant Physiol. 2008;146:5-21.

34. Lynch M, Conery JS. The evolutionary fate and consequences of duplicate genes. Science. 2000;290:1151-5.

35. Juretic N, Hoen DR, Huynh ML, Harrison PM, Bureau TE. The evolutionary fate of MULE-mediated duplications of host gene fragments in rice. Genome Res. 2005:15:1292-7.

36. Castro P, Román B, Rubio J, Die JV. Selection of reference genes for expression studies in Cicer arietinum L.: analysis of cyp81E3 gene expression against Ascochyta rabiei. Mol Breed. 2012;29:261-74.

37. Die JV, Román B. RNA quality assessment: a view from plant qPCR studies. J Exp Bot. 2012;63:6069-77.

38. Schmittgen TD, Livak KJ. Analyzing real-time PCR data by the comparative CT method. Nat Protoc. 2008;3:1101-8.

39. Die JV, Obrero Á, González-Verdejo Cl, Román B. Characterization of the 3":5" ratio for reliable determination of RNA quality. Anal Biochem. 2011;419:336-8.

40. Zuker M. Mfold web server for nucleic acid folding and hybridization prediction. Nucleic Acids Res. 2003;31:3406-15.

41. Die JV, Román B, Nadal S, González-Verdejo Cl. Evaluation of candidate reference genes for expression studies in Pisum sativum under different experimental conditions. Planta. 2010;232:145-53.

42. Vandesompele J, De Preter K, Pattyn F, Poppe B, Van Roy N, De Paepe A, et al. Accurate normalization of real-time quantitative RT-PCR data by geometric averaging of multiple internal control genes. Genome Biol. 2002; 3:RESEARCH0034.

43. Hellemans J, Mortier G, De Paepe A, Speleman F, Vandesompele J. qBase relative quantification framework and software for management and automated analysis of real-time quantitative PCR data. Genome Biol 2007;8:R19.

44. Ramakers C, Ruijter JM, Deprez RHL, Moorman AFM. Assumption-free analysis of quantitative real-time polymerase chain reaction (PCR) data. Neurosci Lett. 2003;339:62-6.

45. Conforte AJ, Guimarães-Dias F, Neves-Borges AC, Bencke-Malato M, FelixWhipps D, Alves-Ferreira $M$, et al. Isolation and characterization of a promoter responsive to salt, osmotic and dehydration stresses in soybean. Genet Mol Biol. 2017;40:226-37.

46. Hernandez-Garcia CM, Finer JJ. Identification and validation of promoters and cis-acting regulatory elements. Plant Sci. 2014;217-218:109-19.

47. Fujimoto SY, Ohta M, Usui A, Shinshi H, Ohme-Takagi M. Arabidopsis ethyleneresponsive element binding factors act as transcriptional activators or repressors of GCC box-mediated gene expression. Plant Cell. 2000;12:393-404.

48. Himmelbach A, Liu L, Zierold U, Altschmied L, Maucher H, Beier F, et al. Promoters of the barley germin-like GER4 gene cluster enable strong transgene expression in response to pathogen attack. Plant Cell. 2010;22: 937-52.

49. Çakir B, Kiliçkaya O, Olcay AC. Genome-wide analysis of Aux/IAA genes in Vitis vinifera: cloning and expression profiling of a grape Aux/IAA gene in response to phytohormone and abiotic stresses. Acta Physiol Plant. 2012;35: 365-77

50. R Core Team. R: a language and environment for statistical computing. Vienna: R Foundation for Statistical Computing; 2017. URL https://www.Rproject.org/

51. RStudio Team. RStudio: integrated development for R. Boston: RStudio, Inc.; 2016. http://www.rstudio.com/

52. Wang D, Pei K, Fu Y, Sun Z, Li S, Liu H, et al. Genome-wide analysis of the auxin response factors (ARF) gene family in rice (Oryza sativa). Gene. 2007; 394:13-24.

53. Wang Y, Deng D, Shi Y, Miao N, Bian Y, Yin Z. Diversification, phylogeny and evolution of auxin response factor (ARF) family: insights gained from analyzing maize ARF genes. Mol Biol Rep. 2012;39:2401-15.

54. Wan S, Li W, Zhu Y, Liu Z, Huang W, Zhan J. Genome-wide identification, characterization and expression analysis of the auxin response factor gene family in Vitis vinifera. Plant Cell Rep. 2014;33:1365-75.
55. Varshney RK, Thudi M, Nayak SN, Gaur PM, Kashiwagi J, Krishnamurthy L, et al. Genetic dissection of drought tolerance in chickpea (Cicer arietinum L.). Theor Appl Genet. 2014;127:445-62.

56. Roosjen $\mathrm{M}$, Paque $\mathrm{S}$, Weijers $\mathrm{D}$. Auxin response factors: output control in auxin biology. J Exp Bot. 2018;69:179-188.

57. Okushima Y, Overvoorde PJ, Arima K, Alonso JM, Chan A, Chang C, et al. Functional genomic analysis of the AUXIN RESPONSE FACTOR gene family members in Arabidopsis thaliana: unique and overlapping functions of ARF7 and ARF19. Plant Cell. 2005;17:444-63.

58. Xing H, Pudake RN, Guo G, Xing G. Genome-wide identification and expression profiling of auxin response factor (ARF) gene family in maize. BMC Genomics. 2011;12:178.

59. Kumar R, Tyagi AK, Sharma AK. Genome-wide analysis of auxin response factor (ARF) gene family from tomato and analysis of their role in flower and fruit development. Mol Gen Genomics. 2011;285:245-60.

60. Yu H, Soler M, Mila I, San Clemente H, Savelli B, Dunand C, et al. Genome-wide characterization and expression profiling of the AUXIN RESPONSE FACTOR (ARF) gene family in Eucalyptus grandis. PLoS One. 2014;9:e108906-13.

61. Mun J-H, Yu H-J, Shin JY, Oh M, Hwang H-J, Chung H. Auxin response factor gene family in Brassica rapa: genomic organization, divergence, expression, and evolution. Mol Gen Genomics. 2012;287:765-84.

62. Zhang J. Evolution by gene duplication: an update. Trends Ecol Evol. 2003; 18:292-8.

63. Shen C, Yue R, Sun T, Zhang L, Xu L, Tie S, et al. Genome-wide identification and expression analysis of auxin response factor gene family in Medicago truncatula. Front Plant Sci. 2015;6:897.

64. Pfeil BE, Schlueter JA, Shoemaker RC, Doyle JJ. Placing Paleopolyploidy in relation to taxon divergence: a phylogenetic analysis in legumes using 39 gene families. Syst Biol. 2005;54:441-54.

65. Li WH, Gojobori T, Nei M. Pseudogenes as a paradigm of neutral evolution. Nature. 1981:292:237-9.

66. Finet C, Fourquin C, Vinauger M, Berne-Dedieu A, Chambrier P, Paindavoine $S$, et al. Parallel structural evolution of auxin response factors in the angiosperms. Plant J. 2010;63:952-9.

67. Kopelman NM, Lancet D, Yanai I. Alternative splicing and gene duplication are inversely correlated evolutionary mechanisms. Nat Genet. 2005;37:588-9.

68. Su Z, Wang J, Yu J, Huang X, Gu X. Evolution of alternative splicing after gene duplication. Genome Res. 2006;16:182-9.

69. EST. Bethesda (MD): National Library of Medicine (US), National Center for Biotechnology Information. 2017. URL https://www.ncbi.nlm.nih.gov/est/. Accessed Nov 2017

70. Wu M-F, Yamaguchi N, Xiao J, Bargmann B, Estelle M, Sang Y, et al. Auxinregulated chromatin switch directs acquisition of flower primordium founder fate elife. 2015:4:e09269.

71. Cherenkov P, Novikova D, Omelyanchuk N, Levitsky V, Grosse I, Weijers D, et al. Diversity of cisregulatory elements associated with auxin response in Arabidopsis thaliana. J Exp Bot. 2018;69:329-339.

72. Spaepen S, Vanderleyden J. Auxin and plant-microbe interactions. Cold Spring Harb Perspect Biol. 2011;3:a001438.

\section{Ready to submit your research? Choose BMC and benefit from:}

- fast, convenient online submission

- thorough peer review by experienced researchers in your field

- rapid publication on acceptance

- support for research data, including large and complex data types

- gold Open Access which fosters wider collaboration and increased citations

- maximum visibility for your research: over $100 \mathrm{M}$ website views per year

At BMC, research is always in progress.

Learn more biomedcentral.com/submissions 